\title{
Impacts of Nanocrystal Location on the Operation of Trap-Layer-Engineered Poly-Si Nanowired Gate-All-Around SONOS Memory Devices
}

\author{
Cheng-Wei Luo, Horng-Chih Lin, Senior Member, IEEE, Ko-Hui Lee, Wei-Chen Chen, \\ Hsing-Hui Hsu, and Tiao-Yuan Huang, Fellow, IEEE
}

\begin{abstract}
Trap-layer-engineered poly-Si nanowire siliconoxide-nitride-oxide-silicon (SONOS) devices with a gate-allaround (GAA) configuration were fabricated and characterized. For the first time, a clever method has been developed to flexibly incorporate Si-nanocrystal (NC) dots in different locations in the nitride layer. Three types of poly-Si GAA SONOS devices with $\mathrm{Si}-\mathrm{NC}$ dots embedded in the block oxide/nitride interface, the middle of the nitride, and the nitride/tunnel oxide interface, respectively, by in situ deposition were fabricated and investigated in this paper. Our results indicate that the optimal NC location appears to be somewhere between the middle and bottom interfaces of the nitride layer.
\end{abstract}

Index Terms-Gate-all-around (GAA), nanocrystal (NC), nanowire (NW), poly-Si, silicon-oxide-nitride-oxide-silicon (SONOS).

\section{INTRODUCTION}

$\mathbf{I}$ $\mathrm{N}$ THE PAST several decades, the floating-gate (FG)-type cell structure has dominated the mainstream nonvolatilesemiconductor-memory market [1]. However, issues such as the strong FG coupling effect between neighboring cells [2] and stress-induced leakage current (SILC) [3] in the tunneling oxide have hindered the future scaling of the FG-type technology. In line of this, the charge-trapping-type cell structure, specifically silicon-oxide-nitride-oxide-silicon (SONOS), has received renewed keen interest for replacing FG Flash cells owing to its excellent immunity to SILC and FG coupling effects. Unlike FG Flash cells, the charges are stored in discrete traps located in the nitride of the SONOS cell [4]; therefore, the tunneling oxide can be further scaled down to allow the lowering of operation voltage and/or improving program/erase (P/E) speed.

Recently, poly-Si-based SONOS device has attracted much attention because of its full process compatibility with 3-D integration [5], [6]. Various approaches have been proposed

Manuscript received December 8, 2010; revised March 3, 2011, March 24, 2011 and March 27, 2011; accepted March 29, 2011. Date of publication April 25, 2011; date of current version June 22, 2011. This work was supported in part by the National Science Council under Contract NSC 96-2221-E-009212-MY3. The review of this paper was arranged by Editor D. Esseni.

C.-W. Luo, K.-H. Lee, W.-C. Chen, H.-H. Hsu, and T.-Y. Huang are with the Department of Electronics Engineering and Institute of Electronics, National Chiao Tung University, Hsinchu 300, Taiwan.

H.-C. Lin is with the Department of Electronics Engineering and Institute of Electronics, National Chiao Tung University, Hsinchu 300, Taiwan, and also with the National Nano Device Laboratories, Hsinchu 300, Taiwan (e-mail: hclin@faculty.nctu.edu.tw).

Color versions of one or more of the figures in this paper are available online at http://ieeexplore.ieee.org.

Digital Object Identifier 10.1109/TED.2011.2140321
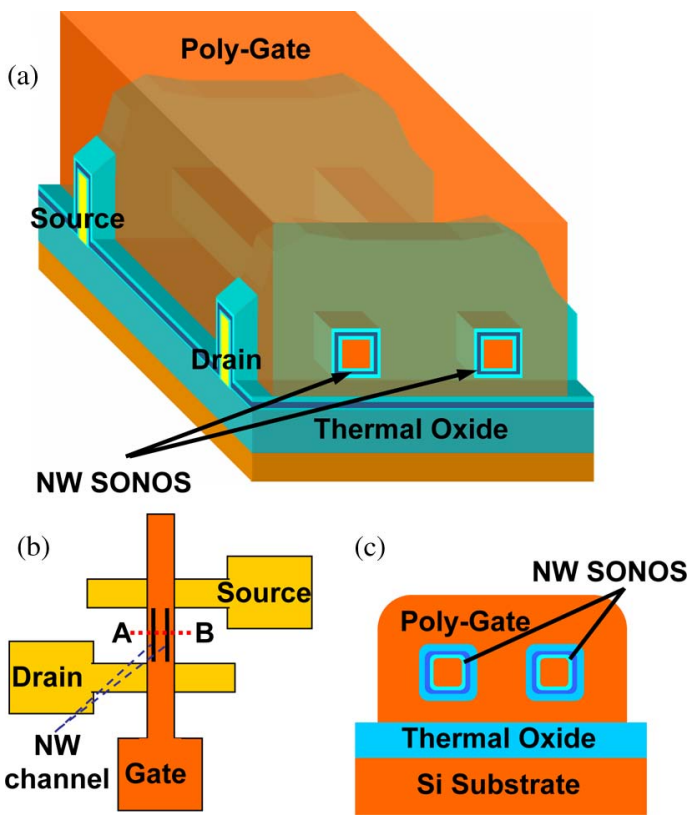

Fig. 1. (a)Schematic structure of the proposed device. (b) Layout of the device. (c) Cross-sectional view of the NW SONOS along line AB in (b).

for improving SONOS device performance such as enhancing gate controllability using multigated schemes such as gate-allaround (GAA) [7], replacing a block oxide layer with a high- $k$ dielectric [8], or adopting a trap-layer-engineering (TLE) scheme [9]-[11]. The concept of TLE was originally proposed by incorporating the tungsten nitride nanodots in the nitride layer. The incorporation of silicon nanocrystal (NC) dots at the top block oxide/nitride interface [10] or in the middle of the nitride [11] has also been proposed and investigated. However, a comprehensive study on the effects of NC-dot locations in a trapping layer on device characteristics is still lacking. In this paper, we have fabricated and investigated several types of poly-Si GAA SONOS devices with Si-NC dots embedded in various locations in the nitride layer by in situ deposition. Particular attention was paid on the effects of the Si-NC-dot location on device operation characteristics.

\section{Device Structures and Fabrication}

Figs. 1(a)-(c) shows the stereo, top, and cross-sectional views, respectively, of the proposed GAA nanowired (NW) device structure. Basically, the fabrication process is similar 


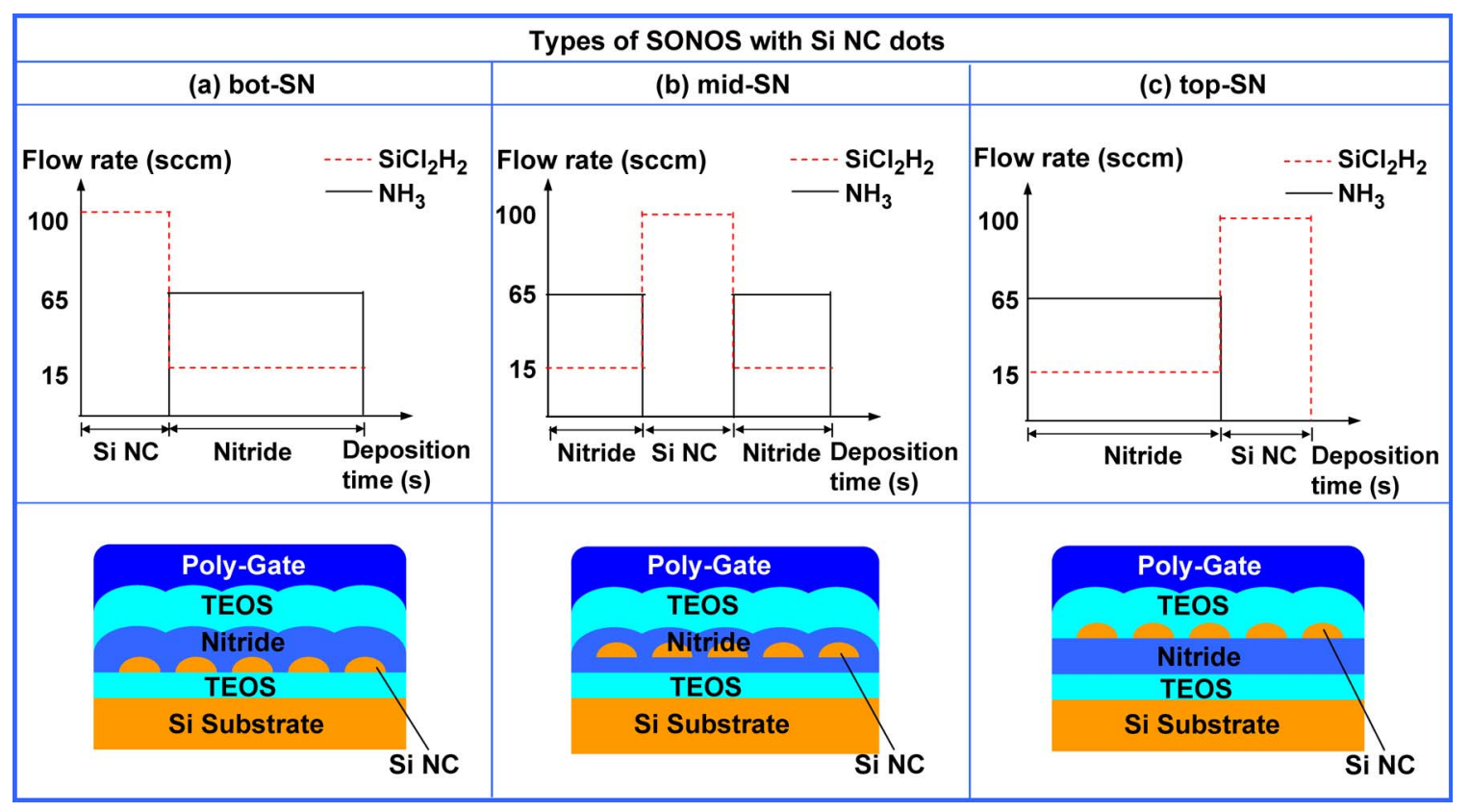

Fig. 2. Flow rate and schematic diagram of three types of devices incorporated with Si-NC dots. Bot-SN: Si-NC dots are located at the interface of the tunneling oxide and the nitride. Mid-SN: Si-NC dots are embedded in the nitride. Top-SN: Si-NCs are located at the interface of the nitride and the block oxide.

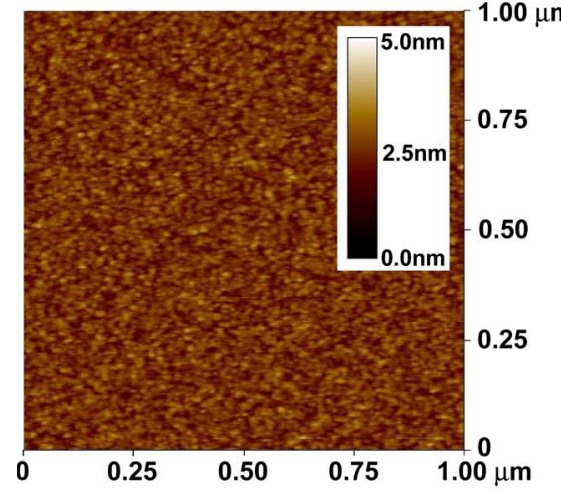

(a)

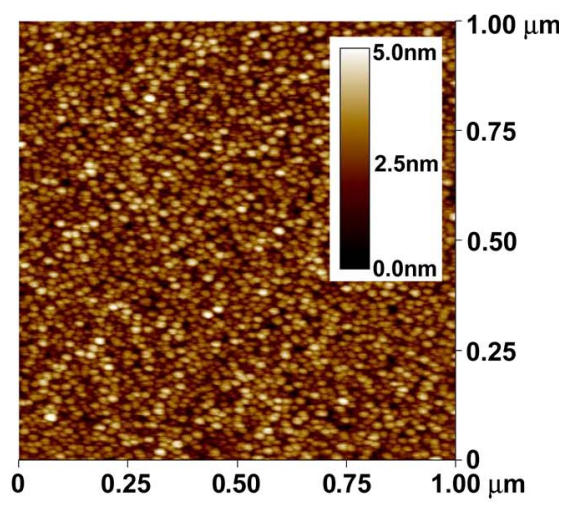

(b)

Fig. 3. $1 \times 1 \mu \mathrm{m}^{2}$ AFM image of nitride films (a) without and (b) with Si-NC dots formed on the surface. The surface roughness (root-mean-square value) is $0.338 \mathrm{~nm}$ in (a) and $0.791 \mathrm{~nm}$ in (b). The sample in (b) has been treated with a DCS exposure time of $45 \mathrm{~s}$, and the density of Si-NC dots is $2.4 \times 10^{11} \mathrm{~cm}^{-2}$.

to that described in one of our previous papers [12], except for the implementation of the TLE scheme by incorporating the Si-NC dots in the nitride layer. Briefly, after forming the silicon NWs, a 3.5-nm tunneling oxide was first deposited, which was followed by nitride deposition with low-pressure chemical vapor deposition (LPCVD). By cleverly adjusting the flow rates of $\mathrm{SiCl}_{2} \mathrm{H}_{2}$ [using a distribution control system (DCS)] and $\mathrm{NH}_{3}$ during the LPCVD-nitride deposition step [see Figs. 2(a)-(c)], the Si-NC dots can be in situ incorporated into various locations in the nitride layer in a flexible and controllable manner. As shown in Figs. 2(a)-(c), wafers were split into three groups, which were denoted as bot-SN, mid-SN, and top-SN, respectively, according to the position of the $\mathrm{Si}$ $\mathrm{NC}$ dots in the nitride. For illustration, we take the mid-SN split shown in Fig. 2(b) as an example. The TLE process consists of three consecutive steps in the LPCVD furnace to form a bottom nitride, $\mathrm{Si}-\mathrm{NC}$ dots, and a top nitride, consecutively. In the first step, a 4-nm-thick bottom nitride film is deposited with DCS (15 sccm) and $\mathrm{NH}_{3}(65 \mathrm{sccm})$ gases at $780{ }^{\circ} \mathrm{C}$. Subsequently, by turning off the $\mathrm{NH}_{3}$ gas flow and increasing the flow rate of the DCS to $100 \mathrm{sccm}, \mathrm{Si}-\mathrm{NC}$ dots are in situ formed on the surface of the deposited nitride. The duration of this period is $45 \mathrm{~s}$. Then, a 4-nm top nitride is capped on the Si-NC dots. Similarly, the gas flow charts for the TLE of bot-SN and top-SN are illustrated in Fig. 2(a) and (c), respectively. After the TLE process, all wafers were combined to receive 12-nmthick tetraethyl orthosilicate (TEOS) oxide deposition as the block oxide. An in situ doped poly Si of about $150 \mathrm{~nm}$ was then deposited and patterned to serve as the gate electrode. For comparison, standard (STD) GAA-NW-SONOS devices with ONO of 3.5/8/12 nm were also fabricated and characterized.

The atomic-force microscopic (AFM) image of nitride samples with and without $\mathrm{Si}-\mathrm{NC}$ dots formed on the surface are shown in Fig. 3(a) and (b), respectively. In contrast to the smooth bare nitride sample, the abundance of protuberant $\mathrm{Si}$ $\mathrm{NC}$ dots with an estimated density of $2.4 \times 10^{11} \mathrm{~cm}^{-2}$ is clearly visible in Fig. 3(b). Note that, for the same process condition forming the NC dots on a TEOS oxide surface, the 


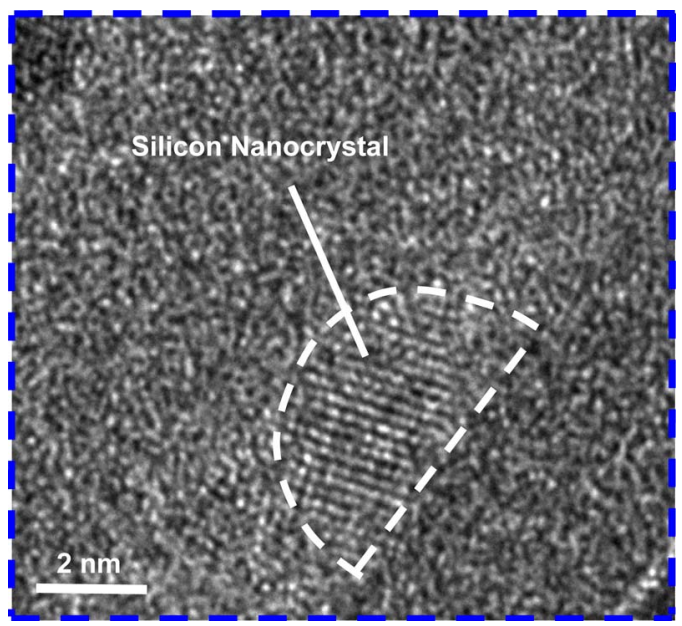

Fig. 4. Cross-sectional TEM picture of a Si-NC dot embedded in the nitride.

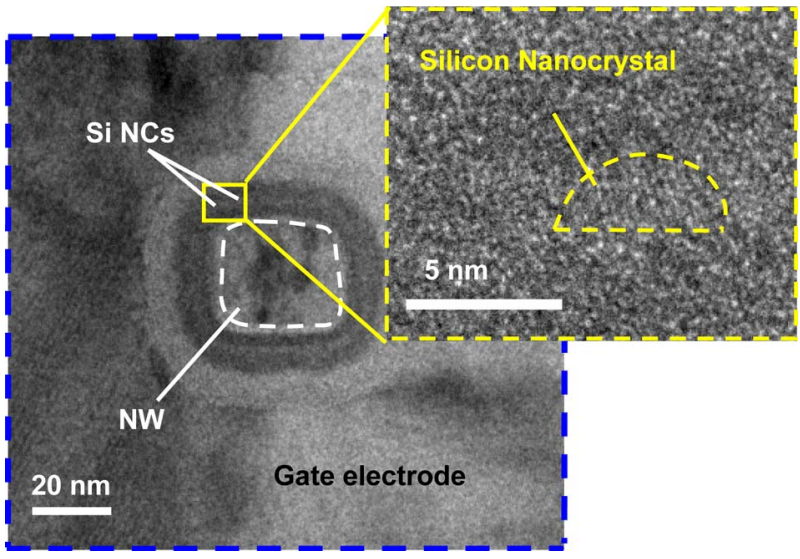

Fig. 5. Cross-sectional TEM picture of a mid-SN device. Inset shows the enlarged view of an embedded Si-NC dot in the nitride.

density is around $2.6 \times 10^{11} \mathrm{~cm}^{-2}$ (data not shown), which is close to the result shown in Fig. 3(b). Fig. 4 depicts a highresolution transmission-electron-microscopic (TEM) image of a test sample showing a hemispherical Si-NC dot formed on the nitride with the aforementioned approach. Fig. 5 exhibits the cross-sectional TEM image of a mid-SN device. It can be seen that the silicon NW is rectangular in shape with rounded corners and the width is about $30 \mathrm{~nm}$. The inset shows an enlarged view of the TLE nitride layer in which a hemispherical Si-NC dot is contained.

\section{BASIC EleCtricAl Characteristics OF POLY-SI NW SONOS DEVICES}

Fig. 6 shows the $I_{D}-V_{G}$ curves of the STD and Si-NC NW SONOS devices with $L=0.4 \mu \mathrm{m}$. It can be seen that the subthreshold swing (SS) of the STD device is slightly lower than those of the Si-NC splits. The discrepancy of the SS can be explained by the following two reasons. First, the embedded $\mathrm{Si}$-NC dots increase the effective oxide thickness (EOT). This is confirmed from the measurements performed on the planar capacitors where the EOT is found to be $18.2 \mathrm{~nm}$ for the STD split and 19.1-19.7 nm for the samples incorporated with NCs. Second, after depositing the $\mathrm{Si} \mathrm{NC}$, the surface is roughened. As

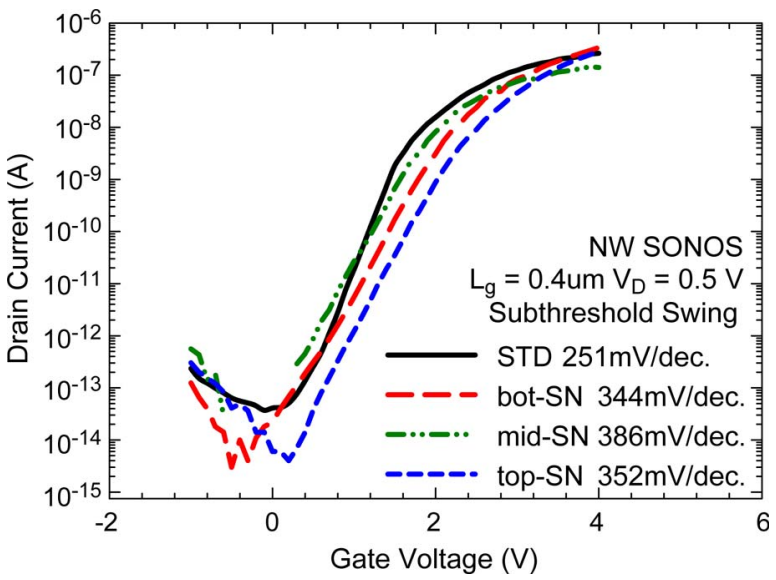

Fig. 6. Transfer characteristics of STD, bot-SN, mid-SN, and top-SN devices, all with a channel length of $0.4 \mu \mathrm{m}$ and measured at $V_{d}=0.1 \mathrm{~V}$.

indicated by the AFM images shown in Fig. 3(a) and (b), the surface roughness (root-mean-square value) indeed increases from $0.338 \mathrm{~nm}$ for the sample without NCs to $0.791 \mathrm{~nm}$ on the sample with NCs formed. Consequently, the EOT of the Si-NC devices is not uniform across all gated area, leading to a nonnegligible variation in EOT across the channel region, which may in turn cause a fluctuation of the channel potential, further degrading the SS. Based on the aforementioned two reasons, it is reasonable to explain why the SS of the STD split is better than those of the Si-NC splits.

\section{Programming/Erasing Characteristics}

For P/E operation, Fowler-Nordheim injection was employed by applying a positive or a negative voltage to the gate electrode for program or erase operation, respectively, with the source and the drain grounded. For the erase tests, all devices were programmed beforehand with a $2.5-\mathrm{V}$ shift of $V_{\text {th }}$ with respect to the fresh state. The program characteristics of the STD device are shown in Fig. 7(a), whereas Fig. 7(b)-(d) depict the program characteristics of the bot-SN, mid-SN, and topSN devices, respectively. Owing to the thinner EOT, the STD device exhibits the fastest $V_{\text {th }}$ increase rate among all splits in the very early stage of programming (e.g., $\ll 10^{-7}$ s). However, when the program time is sufficiently long, both bot-SN [see Fig. 7(b)] and mid-SN [see Fig. 7(c)] devices show faster rates over the STD control. For the STD device, it has been pointed out previously that, during programming, the centroid of the trapped electrons in the nitride layer tends to migrate from the bottom interface toward the middle of the nitride layer [9]. Nevertheless, Si-NC dots in the bot-SN and mid-SN devices provide additional trapping sites in the bottom nitride interface and the nitride center, respectively; therefore, more electrons can be trapped in positions closer to the channel than the STD control during programming. As a result, a larger $V_{\text {th }}$ shift rate and a larger $V_{\text {th }}$ value are resulted in Fig. 7(b) and (c). Furthermore, the available trapping sites also increase due to the incorporation of the Si-NC dots as evidenced by the larger $V_{\text {th }}$ window with prolonged stressing time for both the bot-SN and mid-SN devices. 

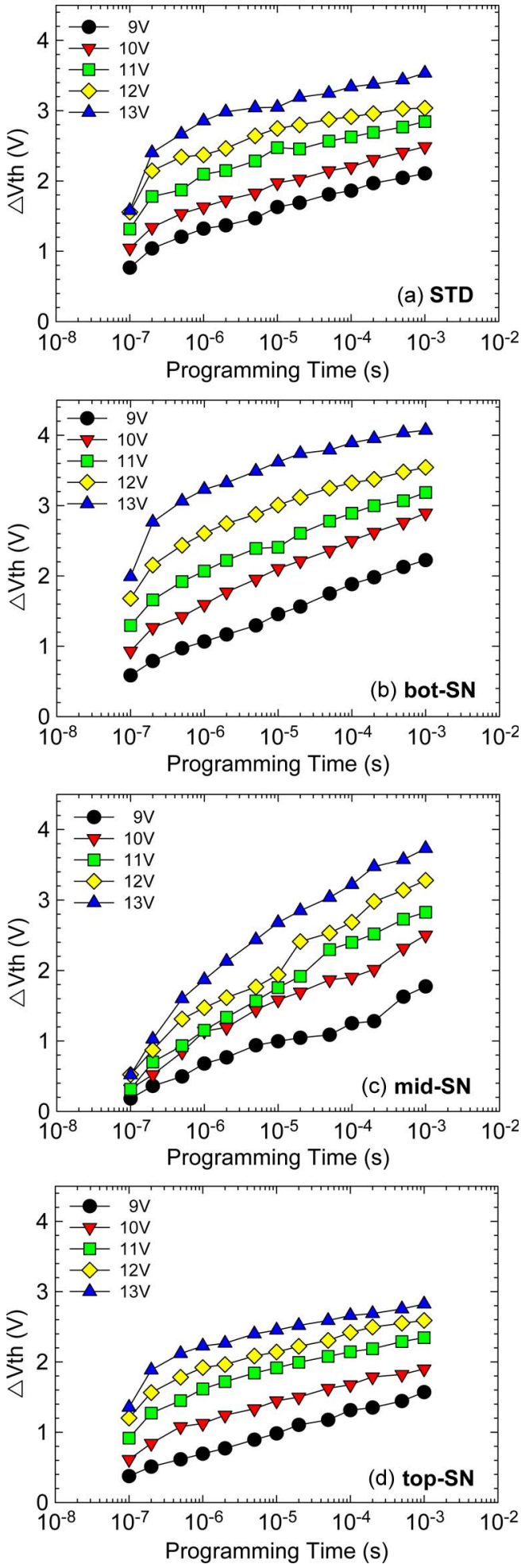

Fig. 7. Program characteristics of (a) STD, (b) bot-SN, (c) mid-SN, and (d) top-SN devices, all with a channel length of $0.4 \mu \mathrm{m}$ and a gate bias that varies from 9 to $13 \mathrm{~V}$.

For the top-SN device, as shown in Fig. 7(d), the $V_{\text {th }}$ shift rate is similar to that of the STD device. The buildup of negative charges in the middle of the nitride during programming may hinder further injection of electrons from the channel; therefore, most of the top Si-NC dots would remain vacant and neutral. Owing to the thicker EOT of the top-SN device, the program

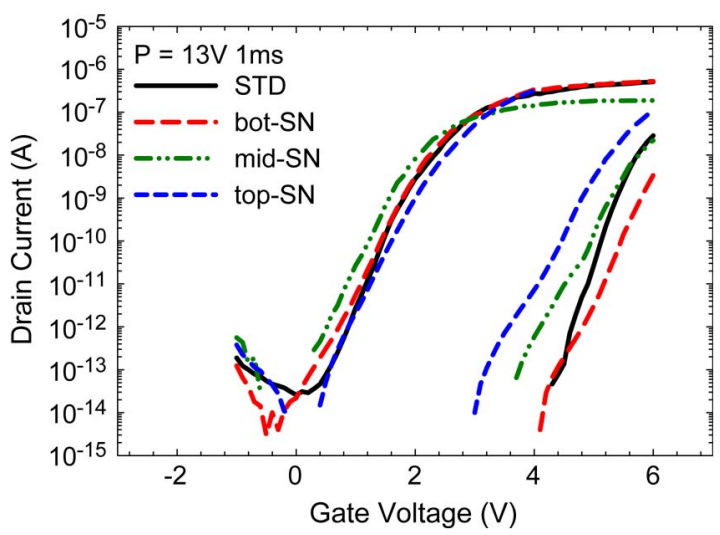

Fig. 8. Transfer characteristics of all splits of devices before and after programming with $V_{g}$ of $13 \mathrm{~V}$ and a stress time of $1 \mathrm{~ms}$.

window is smaller than that of the STD device. Fig. 8 shows the transfer curves of all splits before and after programming operation $\left(V_{g}=+13 \mathrm{~V}\right.$ and $\left.t=10^{-3} \mathrm{~s}\right)$. It can be seen that the bot-SN device exhibits the largest window among all splits.

Fig. 9(a)-(d) shows the erase characteristics of the STD, bot-SN, mid-SN, and top-SN devices, respectively. Compared with the STD device, the bot-SN one shows a faster rate and a mitigated saturation behavior [see Fig. 9(b)]. This is attributed to the fact that more trapped electrons are located closer to the channel. For the mid-SN device [see Fig. 9(c)], the $V_{\text {th }}$ shift rate is initially slow and only weakly dependent on the gate bias (see Stage I in the figure) but becomes much faster at a certain moment, which is closely related to the magnitude of gate bias (i.e., Stage II). Such a transition can be understood with the qualitative band diagrams shown in Fig. 10 and Fig. 11(a) and (b). Fig. 10 shows the situation of a programmed mid$\mathrm{SN}$ device. The Si-NC dots incorporated in the middle of the nitride form quantum wells, which provide trapping sites with an energy level lower than the portion of the trapping sites located inside the nitride. After programming, most of the electrons are believed to be trapped inside the deep levels in the Si-NC dots or the defect sites located in the middle of the nitride, resulting in the rise of potential wherein, as shown in Fig. 10. In Stage I [see Fig. 11(a)] of erase operation, the electrons trapped inside the Si-NC dots can either tunnel back to the channel directly, which would lower $V_{\mathrm{th}}$, or transfer to vacant levels in the nitride, which tend to increase $V_{\mathrm{th}}$. Since the two processes have an opposite effect on the $V_{\mathrm{th}}$ shift and tend to cancel out each other, the overall shift rate is limited. In addition, as the gate bias is varied, the major change in voltage drop occurs in the block oxide due to the accumulation of a large amount of trapped electrons; therefore, the $V_{\text {th }}$ shift rate is not strongly dependent on the gate bias [see Fig. 9(c)]. While in Stage II, most of the electrons trapped in the Si-NC dots have vacated; therefore, the ejection of electrons to the channel from the trapping sites located in the nitride becomes dominant, as shown in Fig. 11(b), resulting in an accelerated rate in the $V_{\text {th }}$ shift shown in Fig. 9(c).

For the top-SN device [see Fig. 9(d)], it exhibits rates comparable to those of the STD one, but $V_{\text {th }}$ begins to shift upwards as the erase time is sufficiently long. This upward trend is explained by the qualitative band diagrams shown in Fig. 12(a) 

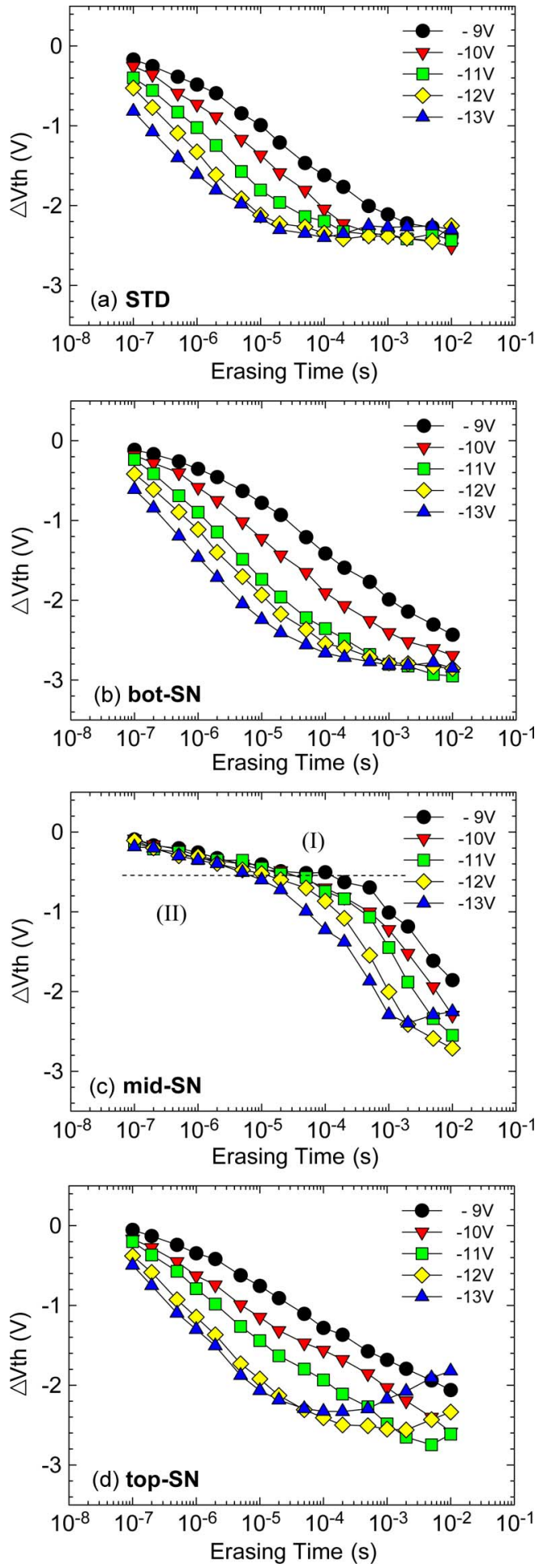

Fig. 9. Erase characteristics of (a) STD, (b) bot-SN, (c) mid-SN, and (d) top$\mathrm{SN}$ devices, all with a channel length of $0.4 \mu \mathrm{m}$ and a gate bias that varies from -9 to $-13 \mathrm{~V}$.

and (b) for the top-SN devices during erase operation. Fig. 12(a) corresponds to the early stage of operation during which the trapped electrons are released to the channel, resulting in $V_{\text {th }}$ lowering. Later, as shown in Fig. 12(b), as most of the trapped electrons stored in the nitride have vacated, the field strength in the block oxide will increase, causing electron injection from the gate. The injected electrons then get trapped in the Si-NC dots and cause a $V_{\mathrm{th}}$ increase.

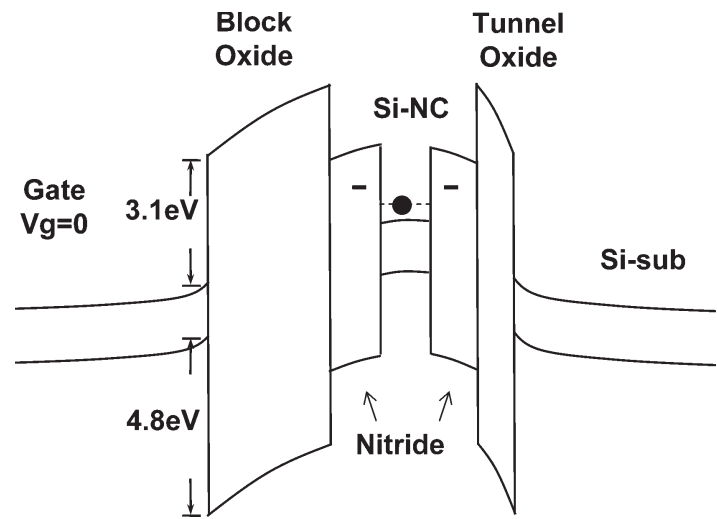

Fig. 10. Band diagrams of the programmed mid-SN device.
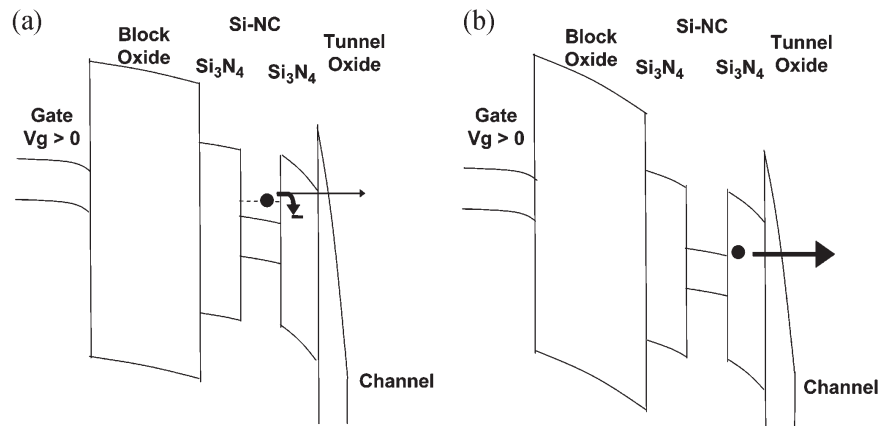

Fig. 11. Band diagrams of the mid-SN device during erasing at (a) Stages I and (b) II. At Stage 1, the electrons trapped inside the Si-NC dots can either tunnel back to the channel directly or transfer to a vacant level in the nitride.
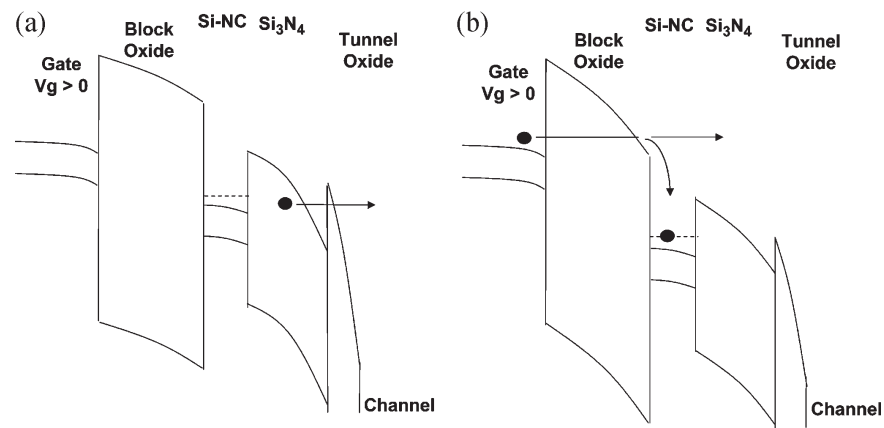

Fig. 12. Band diagrams of a top-SN device during erasing. (a) Electrons are detrapped from the nitride layer. (b) Electrons injected from the gate as most of the electrons stored in the nitride have vacated. Some injected electrons are trapped in the Si-NC dots.

\section{ENDURANCE AND RETENTION CHARACTERISTICS}

For the reliability tests, different $\mathrm{P} / \mathrm{E}$ conditions were applied to different splits in order to obtain the same memory window of about $2 \mathrm{~V}$ for all splits. Fig. 13 shows the retention characteristics of all splits measured at $25^{\circ} \mathrm{C}$ and $85^{\circ} \mathrm{C}$. It can be seen that the mid-SN split shows the best retention performance among all splits. This is believed to be due to the deeper storage levels for electrons introduced by $\mathrm{Si}-\mathrm{NC}$ dots embedded in the nitride. In contrast, the bot-SN device exhibits a retention behavior similar to that of the STD control. This is because the trapped electrons tend to leak out because, compared with other splits, they are located closer to the channel, although the incorporated Si-NC dots do provide deeper levels for storing the electrons. Finally, the endurance characteristics for all splits are 


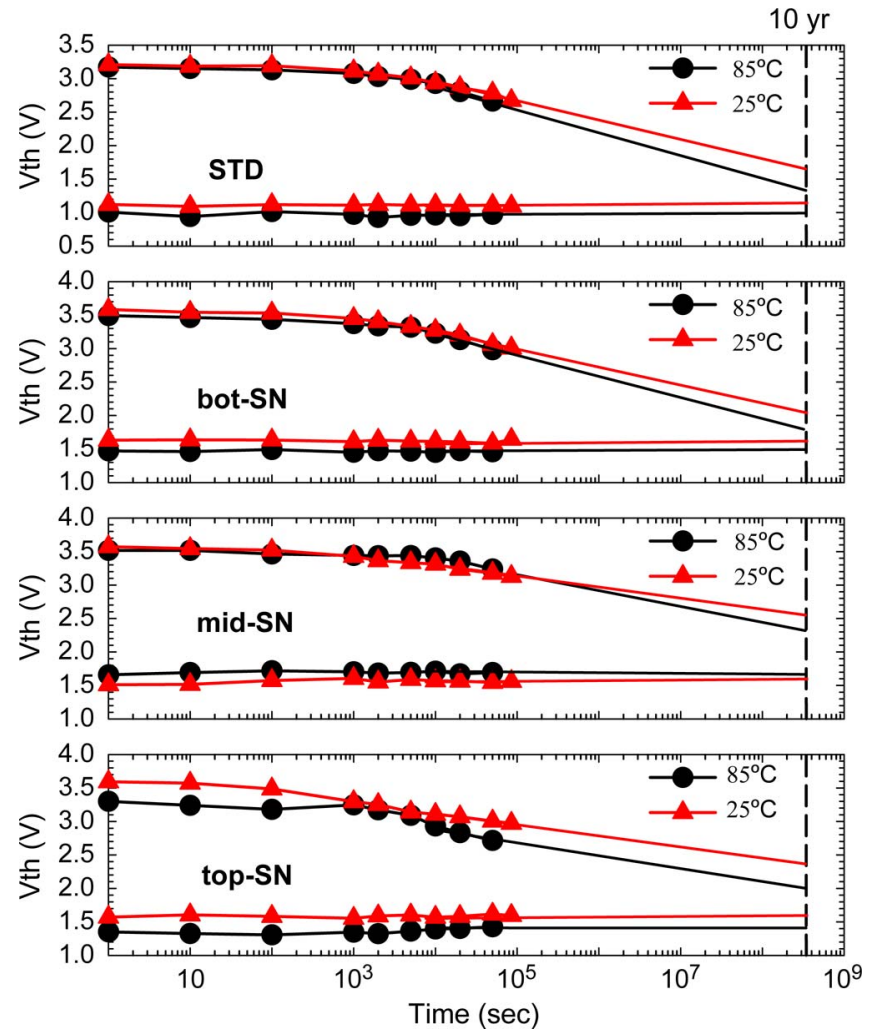

Fig. 13. Data retention characteristics of the STD device and various Si-NC devices. The memory window is initially set at about $2 \mathrm{~V}$ for all devices.

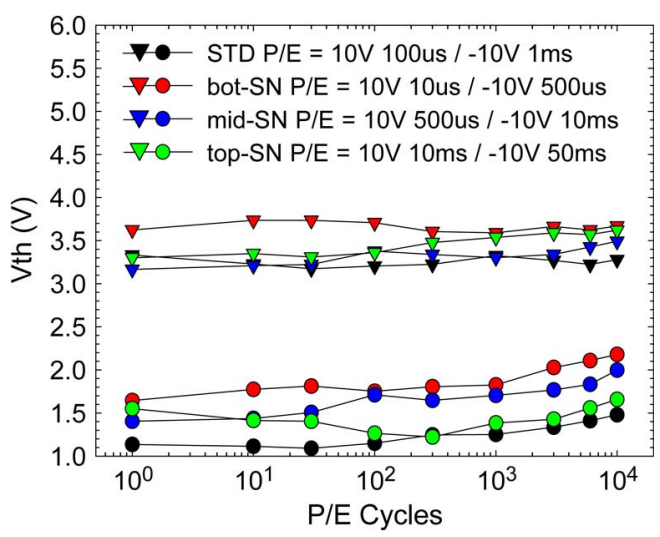

Fig. 14. Endurance characteristics of the STD device and various Si-NC devices.

TABLE I

COMPARISON BETWEEN THE STD AND Si-NC DEVICES

$\star$ Excellent $\circ$ Good $\triangle$ Fair.

\begin{tabular}{|c|c|c|c|c|}
\hline & STD & bot-SN & mid-SN & top-SN \\
\hline Program Rate $\left(>10^{-7} \mathbf{s}\right)$ & $\bigcirc$ & $\star$ & $\triangle$ & $\triangle$ \\
\hline Erase Rate & $\bigcirc$ & $\star$ & $\triangle$ & $\triangle$ \\
\hline Erase Saturation & $\bigcirc$ & $\star$ & $\bigcirc$ & $\triangle$ \\
\hline Window Size & $\triangle$ & $\star$ & $\bigcirc$ & $\triangle$ \\
\hline Retention & $\triangle$ & $\triangle$ & $\star$ & $O$ \\
\hline Endurance & $\bigcirc$ & $\bigcirc$ & $\bigcirc$ & $O$ \\
\hline
\end{tabular}

shown in Fig. 14. It can be seen that no significant differences are observed among the devices. A comparison of the operation performance of all splits is summarized in Table I.

\section{CONCLUSION}

In this paper, three types of poly-Si GAA-NW-SONOS devices with $\mathrm{Si}-\mathrm{NC}$ dots embedded in the block oxide/nitride interface, middle of the nitride, and tunnel oxide/nitride interface, respectively, have been successfully fabricated and carefully characterized to gain insights into the effects of NC-dot locations on the device memory characteristics. We found that the memory devices exhibit drastic different $\mathrm{P} / \mathrm{E}$ characteristics in accord with their Si-NC-dot locations. During programming, the mean location of trapped electrons would be affected by $\mathrm{Si}$-NC dots, thus inducing a faster $V_{\text {th }}$ increase rate in the bot-SN split. Moreover, these Si-NC dots provide extra trapping sites that enable a larger memory window in both bot-SN and mid-SN splits. While for the mid-SN devices, we found that these Si-NC dots render the trapped electrons harder to detrap. With prolonged erasing time, the gate-injected electrons are trapped in the Si-NC dots, resulting in a $V_{\text {th }}$ increase as is observed in the top-SN devices. To summarize, the bot-SN split shows the best P/E characteristics, whereas the mid-SN split shows the best retention characteristics. Based on these findings, we conclude that the most optimal NC-dot location should be somewhere between the middle and the bottom interface of the nitride layer.

\section{ACKNOWLEDGMENT}

The authors would like to thank the staff of National Nano Device Laboratories for assistance in device fabrication.

\section{REFERENCES}

[1] S. Lai, "Non-volatile memory technologies: The quest for ever lower cost," in IEDM Tech. Dig., Dec. 2008, pp. 11-16.

[2] D. Kang, S. Jang, K. Lee, J. Kim, H. Kwon, W. Lee, B. G. Park, J. D. Lee, and H. Shin, "Improving the cell characteristics using low-k gate spacer in 1G NAND flash memory," in IEDM Tech. Dig., Dec. 2006, pp. 1001-1004.

[3] R. Bez, E. Camerlenghi, A. Modelli, and A. Visconti, "Introduction to flash memory," Proc. IEEE, vol. 91, no. 4, pp. 489-502, Apr. 2003.

[4] M. H. White, D. A. Adams, and B. Jiankang, "On the go with SONOS," IEEE Circuits Devices Mag., vol. 16, no. 4, pp. 22-31, Jul. 2000.

[5] R. Katsumata, M. Kito, Y. Fukuzumi, M. Kido, H. Tanaka, Y. Komori, M. Ishiduki, J. Matsunami, T. Fujiwara, Y. Nagata, L. Zhang, Y. Iwata, R. Kirisawa, H. Aochi, and A. Nitayama, "Pipe-shaped BiCS flash memory with 16 stacked layers and multi-level-cell operation for ultra high density storage devices," in VLSI Symp. Tech. Dig., 2009, pp. 136-137.

[6] J. Jang, H. S. Kim, W. Cho, H. Cho, J. Kim, S. I. Shim, Y. Jang, J. H. Jeong, B. K. Son, D. W. Kim, Kihyun, J. J. Shim, J. S. Lim, K. H. Kim, S. Y. Yi, J. Y. Lim, D. Chung, H. C. Moon, S. Hwang, J. W. Lee, Y. H. Son, U. I. Chung, and W. S. Lee, "Vertical cell array using TCAT (Terabit Cell Array Transistor) technology for ultra high density NAND flash memory," in VLSI Symp. Tech. Dig., 2009, pp. 192-193.

[7] J. Fu, N. Singh, K. D. Buddharaju, S. H. G. Teo, C. Shen, Y. Jiang, C. Zhu, M. B. Yu, G. Q. Lo, N. Balasubramanian, D. L. Kwong, E. Gnani, and G. Baccarani, "Si-nanowire based gate-all-around nonvolatile SONOS memory cell," IEEE Electron Device Lett., vol. 29, no. 5, pp. 518-521, May 2008.

[8] C. H. Lee, K. I. Choi, M. K. Cho, Y. H. Song, K. C. Park, and K. Kim, "A novel SONOS structure of $\mathrm{SiO}_{2} / \mathrm{SiN} / \mathrm{Al}_{2} \mathrm{O}_{3}$ with TaN metal gate for multi-giga bit flash memories," in IEDM Tech. Dig., 2003, pp. 613-616.

[9] Y. J. Ahn, J. D. Choe, J. J. Lee, D. Choi, E. S. Cho, B. Y. Choi, S. H. Lee, S. K. Sung, C. H. Lee, S. H. Cheong, D. K. Lee, S. B. Kim, D. Park, and B. I. Ryu, "Trap layer engineered FinFET NAND flash with enhanced memory window," in VLSI Symp. Tech. Dig., 2006, pp. 88-89. 
[10] J. Fu, K. D. Buddharaju, S. H. G. Teo, C. Zhu, M. B. Yu, N. Singh, G. Q. Lo, N. Balasubramanian, and D. L. Kwong, "Trap layer engineered gate-all-around vertically stacked twin Si-nanowire nonvolatile memory," in IEDM Tech. Dig., 2007, pp. 79-82.

[11] T. Y. Chiang, T. S. Chao, Y. H. Wu, and W. L. Yang, "High-program/erasespeed SONOS with in-situ silicon nanocrystals," IEEE Electron Device Lett., vol. 29, no. 10, pp. 1148-1151, Oct. 2008.

[12] H. H. Hsu, T. W. Liu, L. Chan, C. D. Lin, T. Y. Huang, and H. C. Lin, "Fabrication and characterization of multiple-gated poly-Si nanowire thin-film transistors and impacts of multiple-gate structures on device fluctuations," IEEE Trans. Electron Devices, vol. 55, no. 11, pp. 3063-3069, Nov. 2008.

[13] H. T. Lue, P. Y. Du, S. Y. Wang, K. Y. Hsieh, R. Liu, and C. Y. Lu, "A study of gate-sensing and channel-sensing (GSCS) transient analysis method-Part I: Fundamental theory and applications to study of the trapped charge vertical location and capture efficiency of SONOS-type devices," IEEE Trans. Electron Devices, vol. 55, no. 8, pp. 2218-2228, Aug. 2008.

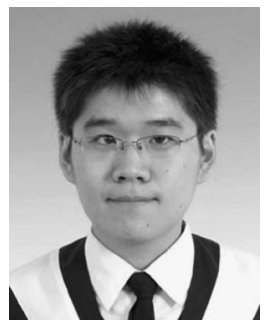

Cheng-Wei Luo was born in Taipei, Taiwan, in 1986. He received the B.S. degree in electrical engineering from National Central University, Taoyuan, Taiwan, in 2008 and the M.S. degree in electronics engineering from National Chiao Tung University, Hsinchu, Taiwan, in 2010

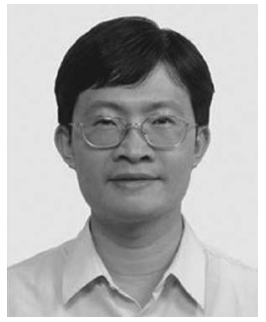

Horng-Chih Lin (S'91-M'95-SM'01) was born in I-Lan, Taiwan, on August 1, 1967. He received the B.S. degree from National Central University, Chung-Li, Taiwan, in 1989, and the Ph.D. degree from National Chiao Tung University (NCTU), Hsinchu, Taiwan, in 1994.

From 1994 to 2004, he was with the National Nano Device Laboratories, where he has been engaged in the research projects of nanoscale device technology development. In 2004, He joined the faculty of NCTU, and since 2007, he has been a Professor with the Department of Electronics Engineering and the Institute of Electronics, NCTU. He is the author or coauthor of over 200 technical papers in international journals and conferences. His current research interests include thin-film transistor fabrication and characterization, reliability of complementary metal-oxide-semiconductor devices, and nanowire device technology.

Dr. Lin was a member of the Program Committee of the International Reliability Physics Symposium in 2001 and 2002 and the International Conference on Solid-State Devices and Materials in 2005-2008.

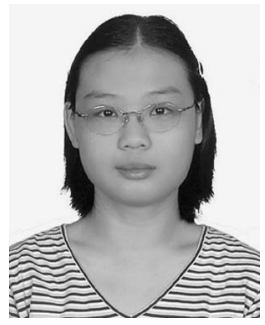

Ko-Hui Lee was born in Taipei, Taiwan, in 1983. She received the B.S. degree from National Chung Cheng University, Chiai, Taiwan, in 2005, and the M.S. degree from National Chiao Tung University (NCTU), Hsinchu, Taiwan, in 2007. She is currently working toward the Ph.D. degree with the Department of Electronics Engineering and Institute of Electronics, NCTU.

Her major research interests are in the field of Si nanowire field-effect transistor devices and nanocrystal memory devices.

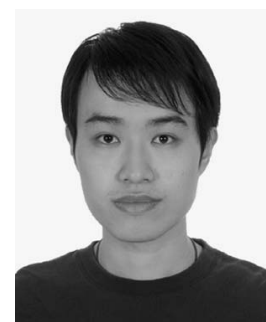

Wei-Chen Chen was born in Taoyuan, Taiwan, in 1984. He received the B.S. degree in electrophysics from National Chiao Tung University (NCTU), Hsinchu, Taiwan, in 2006. He is currently working toward the Ph.D. degree with the Institute of Electronics, NCTU.

His current research interests include the fabrication and characterization of nanowire transistors and germanium-based devices.

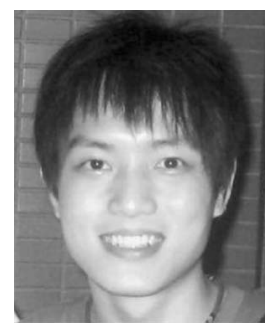

Hsing-Hui Hsu was born in Taipei, Taiwan, in 1982. $\mathrm{He}$ received the B.S. degree in electrical engineering from National Taiwan University, Taipei, Taiwan in 2004 and the M.S. degree from the National ChiaoTung University (NCTU), Hsinchu, Taiwan, in 2008. $\mathrm{He}$ is currently working toward the Ph.D. degree with the Institute of Electronics Engineering, NCTU.

His current research interests include the fabrication and characterization of Si nanowire field-effect transistors and memory devices.

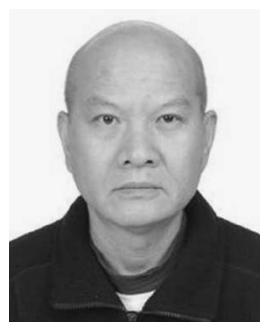

Tiao-Yuan Huang (S'78-M'78-SM'88-F'95), received the B.S.E.E. and M.S.E.E. degrees from National Cheng Kung University, Tainan, Taiwan, in 1971 and 1973, respectively, and the Ph.D. degree from the University of New Mexico, Albuquerque, $\mathrm{NM}$, in 1981.

In 1977, after serving two years in the Taiwanese Navy as fulfillment of his conscription duty, he left for the U.S. He has worked in the U.S. semiconductor industry for 14 years prior to his return to Taiwan. Since 1995, he has been a professor with the Department of Electronics Engineering, National Chiao Tung University, Hsinchu, Taiwan.

Dr. Huang was the recipient of the Semiconductor International's Technology Achievement Award for his invention and demonstration of the fully overlapped lightly doped drain metal-oxide-semiconductor transistors. 